\title{
Depletable Channels: Dynamics and Behaviour
}

\author{
Pietro Cenciarelli, Daniele Gorla and Ivano Salvo \\ University of Rome, "La Sapienza", Dpt. of Computer Science \\ \{cenciarelli,gorla, salvo\} @ di.uniroma1.it
}

\begin{abstract}
A simple model of multi-hop communication in ad-hoc networks is considered. Similar models are often adopted for studying energy efficiency and load balancing of different routing protocols. We address an orthogonal question never considered by the networking community: whether, regardless of specific protocols, two networks may be considered as equivalent from the viewpoint of the communication service they provide. In particular, we consider equivalent two networks with identical maximum and minimum inhibiting flow, and prove that this notion of equivalence coincides with a standard trace-based notion of equivalence borrowed from the theory of concurrency. We finally study the computational complexity of the proposed equivalence and discuss possible alternatives.
\end{abstract}

\section{Introduction}

In recent years, much attention has been devoted to research in the area of ad hoc networking. Many complex theoretical problems are at stake and a variety of efficient routing protocols have been studied for exchanging information across a network without using centralized control $[25,24,6]$.

Ad hoc networks are typically wireless, and multi-hop communication is adopted because of limited wireless transmission range. Moreover, they usually exhibit dynamic behaviour in that their topology may vary over time as a result of mobility or resource consumption. In particular, a crucial kind of resource in most sensor network applications is energy $[3,4]$.

In this paper we study the dynamics of ad hoc communication in a rather simple, and yet significant network model. Dynamics is meant in the sense of change of state and is induced by energy consumption. Similar models have been adopted for studying energy efficiency and load balancing of different routing protocols $[10,18]$. Here we address an orthogonal question which has not received attention in the literature on computer networks as yet: whether, regardless of specific protocols, two networks may be considered as equivalent from the viewpoint of the communication service they provide.

In our framework, a network is a (possibly cyclic) oriented graph equipped with a function associating with each node a non-negative integer representing depletable charge. We are interested in networks as channels for transmitting information. Thus, we consider communication channels, i.e. networks with a chosen pair of nodes called source and target. At a given time a number of 
$(\eta)$

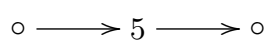

$(\zeta)$

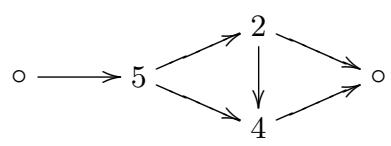

$(\theta)$

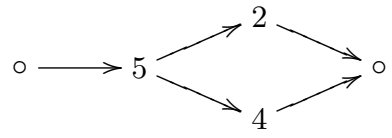

$(\xi)$

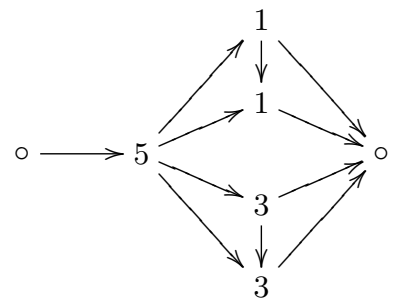

Figure 1. Four channels

atomic items are fed to the source and instantly flow to the target. Charges may change as result of information passing through the net. Each item passing through a node consumes one unit of the node's charge, thus leaving the channel in a state of lower energy.

In drawing channels, we let $n$ stand for a node of charge $n$. Circles (o) are used to denote nodes whose charge is large enough to be irrelevant. Source and target are drawn respectively as the leftmost and rightmost node in the picture. Four channels are depicted in Figure 1. When three items are transmitted along channel $\theta$, node charges may change as shown below.

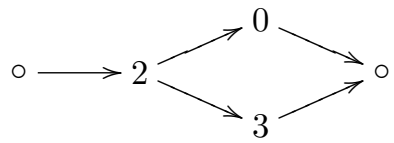

In particular, this result is obtained by routing two items along the northern path, $\circ 52 \circ$, and one along the southern, $\circ 54 \circ$. In this new state the channel is still capable of transmitting two more items, after which the channel is dead, i.e. any transmission from source to target is inhibited by some exhausted node. Different routings of three items are also possible in $\theta$ : for example, letting them all pass south. On the other hand, not all of them may choose the northern path, as its capacity is limited to 2 by the upper node. For the same reason $\theta$ can only transmit up to five items, which is the value of its minimum cut. Indeed, all four channels in the picture support a maximum flow of 5 ; but are they all equivalent?

In our model, where fault tolerance is not at stake, we may intuitively agree that $\eta$ and $\theta$ are indistinguishable in the source-to-target communication service they provide. For example, we could view $\eta$ as the specification of a communication service and $\theta$ as a possible implementation. However, would $\zeta$ implement $\eta$ correctly? We argue that the two channels may behave differently: while $\eta$ and $\theta$ are always alive after any transmission of four items, not so for $\zeta$, where the 4 -valued flow sending two items along the path $\circ 524 \circ$ and two along $\circ 54 \circ$ yields a dead channel. Similarly, channel $\xi$ may be killed by a flow of just four 
items. Then again: can $\zeta$ and $\xi$ be considered as different implementations of the same communication service?

The present paper moves a first step towards a formal study of energysensitive network behaviour. We study a natural notion of network equivalence which equates $\eta$ with $\theta$ of Figure 1 , but not with $\zeta$ and $\xi$. In particular, we shall equate two nets with identical maximum flow and minimum inhibiting flow (i.e., the minimum number of items whose transmission leads to a dead channel). Such an equivalence has a well known corresponding notion in the theory of concurrency, i.e. it corresponds to (complete) trace equivalence [1] built up over the labeled transition system arising from all the possible transmissions a channel can be engaged in. More refined notions of behavioural equivalence are studied in concurrency; most notably bisimulation $[19,16]$. We show that, in spite of its simplicity, our model exhibits a variety of natural notions of behavioural equivalences, whose richness is comparable with that of process calculi. In particular, we shall reveal in Section 5 that, although trace equivalent, $\zeta$ and $\xi$ in Figure 1 do exhibit different behaviour and can in fact be distinguished in terms of bisimulation.

We believe that a theory of behavioural equivalence relating different network topologies and charge distributions may provide guidance in solving optimization problems and a better understanding of protocol properties, such as invariance with respect to sameness of behaviour.

The paper is structured as follows. First, we present our model of energysensitive network channels in Section 2, by describing a simple graph-based model and the associated dynamic behaviours expressed in terms of labeled transitions from a channel to another one. Then, in Section 3, we define a notion of channel equivalence by means of intrinsic features of channels; we then relate such an equivalence to a standard trace-based equivalence built up over the transitions previously defined. This is the main result of the paper. Section 4 tackles complexity issues and shows that trace equivalence is a computationally hard problem, even when restricting to acyclic networks. Section 5 relates trace equivalence with bisimulation. Section 6 discusses related work and draws conclusions.

\section{The Model}

An oriented graph $(V, E)$ consists of a set $V$ of vertices (or nodes) and a set $E \subseteq V \times V$ of ordered pairs of vertices, called edges. A walk is a sequence $u_{1} \ldots u_{n}$ of nodes such that $\left(u_{i}, u_{i+1}\right)$ is an edge, for all $i<n$. We call network a finite oriented graph equipped with a function $\eta$ associating with each node a non-negative integer representing depletable charge. We write just $\eta$ to denote a network $(V, E, \eta)$ when its underlying graph, called topology, is understood.

We study networks as means to transmit information. Once fixed a source node, written $s$, and a target node, written $t$, we call the network a communication channel (channel for short). A path in a channel is a (possibly cyclic) directed walk from $s$ to $t$. The set of paths of a channel $\eta$ is denoted by $P(\eta)$. 
We write $u \stackrel{p}{\sim} v$ to specify that the first and last nodes of a path $p$ are $u$ and $v$ respectively; $u \rightsquigarrow v$ denotes such a path when the name $p$ is not relevant. If $p$ is a walk of the form $u \rightsquigarrow v \rightsquigarrow v^{\prime} \rightsquigarrow w$, we denote by $v \stackrel{p}{\rightsquigarrow} v^{\prime}$ the portion of $p$ from $v$ to $v^{\prime}$.

Paths are often defined in the literature as acyclic walks, and path-oriented definitions of flow associate numerical values separately to paths and cycles [2, Section 3.5]. In our framework, this would amount to allowing spontaneous flow, not originating in the source and depleting the network by cycling in it without ever reaching the target. Since we are interested in modeling information traveling the net as result of a communication act by $s$, we restrict our attention to flows from $s$ to $t$. Formally: let $r_{v p}$ denote the number of times in which a node $v$ is repeated in a path $p$ (zero if $v \notin p$ ); A flow for $\eta$ is a function $\phi: P(\eta) \rightarrow \mathbb{N}$ such that $\phi(v) \leq \eta(v)$, for every $v \in V$, where $\phi(v)$ denotes the amount of $v$ 's charge consumed by $\phi$, that is $\phi(v)=\sum_{p \in P(\eta)} r_{v p} \cdot \phi(p)$. The value of $\phi$ is $\sum_{p \in P(\eta)} \phi(p)$. We denote by $\max _{\eta}$ the value of the maximum flow for $\eta$. We call $\eta$ a dead channel if $\max _{\eta}=0$.

To capture the notion of channel dynamics, we introduce a labeled transition relation $\longrightarrow$ over channels of identical topology, where $(V, E, \eta) \stackrel{n}{\longrightarrow}(V, E, \theta)$ is defined to hold when there exists a flow $\phi$ of value $n$ in $\eta$ such that $\theta(v)=$ $\eta(v)-\phi(v)$ for all nodes $v$. The flow $\phi$ is said to witness the transition. A transition $\eta \stackrel{n}{\longrightarrow} \theta$, and likewise any witness of it, is said to inhibit $\eta$ if $\theta$ is dead. We denote by $\min _{\eta}$ the smallest value of an inhibiting flow in $\eta$.

To conclude, we now give two simple properties of the labeled transition system just defined, namely composition and decomposition of transitions.

Proposition 1. If $\eta \stackrel{n}{\longrightarrow} \theta \stackrel{m}{\longrightarrow} \zeta$ are transitions, so is also $\eta \stackrel{n+m}{\longrightarrow} \zeta$.

Proof. Let $\phi$ and $\psi$ witness the two transitions above. It is easy to check that the function assigning $\phi(p)+\psi(p)$ to each path $p$ of $\eta$ is indeed a flow of value $n+m$.

Proposition 2. If $\eta \stackrel{n+m}{\longrightarrow} \zeta$ is a transition, so are $\eta \stackrel{n}{\longrightarrow} \theta \stackrel{m}{\longrightarrow} \zeta$, for some $\theta$.

Proof. It is sufficient to show it for $m=1$; the general result follows from Proposition 1. Let $\phi$ witness the $n+1$ transition and let $p$ be a path with $\phi(p) \geq 1$. The function assigning $\phi(q)$ to all paths $q \neq p$ and $\phi(p)-1$ to $p$ is clearly a flow witnessing a transition $\eta \stackrel{n}{\rightarrow} \theta$, while $\theta \stackrel{1}{\rightarrow} \zeta$ is obtained by the flow assigning 1 to $p$ and 0 to all other paths.

\section{Behavioural Equivalence}

Two channels may be indistinguishable in the service they provide; such are $\eta$ and $\theta$ of Figure 1. This statement can be made precise by equipping our model with a notion of channel behaviour, so that channels exhibiting identical behaviour may be considered as different implementations of the same communication service. To that effect, we first identify the observations an external user is allowed to 
make on a channel. This establishes the level of abstraction at which channels may be distinguished.

The very first attempt one can do in this direction is to equate all channels with the same maximum flow. In this way, we would equate two channels by only considering an intrinsic (or structural) property of the equated channels, without looking at their dynamic behaviour that arises from the transitions defined for our model. However, it is possible to bridge the structural view put forward by the maximum flow and the dynamic behaviour arising from the transitions. Indeed, as a first theoretical result of this paper, we prove that this structural property of the channel has a well-known counterpart in concurrency theory: it corresponds to what is usually called general trace equivalence for labeled transition systems [1]. By straightforwardly adapting the standard definitions to our framework, a general trace for a channel $\eta$ is a sequence $\left\langle n_{1} \ldots n_{k}\right\rangle$ such that there exist transitions $\eta_{0} \stackrel{n_{1}}{\longrightarrow} \eta_{1} \ldots \stackrel{n_{k}}{\longrightarrow} \eta_{k}$ where $\eta_{0}=\eta$.

Lemma 1. For every $\eta$ and $n \leq \max _{\eta}$, there exists $\eta^{\prime}$ such that $\eta \stackrel{n}{\rightarrow} \eta^{\prime}$.

Proof. By a straightforward induction on $n$ and standard results of flow networks.

Theorem 1. Two channels have identical maximum flow if and only if they have identical sets of general traces.

Proof. (If) By contradiction: assume, e.g., that $\max _{\eta}<\max _{\zeta}=n$. Then, there exists $\zeta^{\prime}$ such that $\zeta \stackrel{n}{\rightarrow} \zeta^{\prime}$. However, there exists no $\eta^{\prime}$ such that $\eta \stackrel{n}{\rightarrow} \eta^{\prime}$; contradiction.

(Only if) Let $\max _{\eta}=\max _{\zeta}$ and let $\left\langle n_{1} \ldots n_{k}\right\rangle$ be a general trace of $\eta$. By Proposition $1, \eta \stackrel{n}{\rightarrow} \eta^{\prime}$, for some $\eta^{\prime}$ and $n=n_{1}+\ldots+n_{k}$. Since $n \leq \max _{\eta}=\max _{\zeta}$, by Lemma $1 \zeta \stackrel{n}{\rightarrow} \zeta^{\prime}$, for some $\zeta^{\prime}$. By Proposition 2 , we conclude that $\left\langle n_{1} \ldots n_{k}\right\rangle$ is a general trace of $\zeta$.

In this way, we would equate all the channels in Figure 1: they all have a maximum flow of value 5 . In particular, every net $\eta$ with $n=\max _{\eta}$ is equivalent to the net

$$
\circ \rightarrow n \rightarrow \circ
$$

However, as noticed in the introduction, $\zeta$ and $\xi$ can be distinguished from $\eta$ and $\theta$ by observing death. Since users $d o$ notice when channels are dead, we seek a more refined notion of equivalence capable of distinguishing $\zeta$ and $\xi$ from $\eta$ and $\theta$.

To this aim, we can also consider the smallest value of an inhibiting flow, viz. $\min _{\eta}$. We can now equate two channels that have the same maximum and minimum inhibiting flow value. In this way, channels $\eta$ and $\theta$ of Figure 1 would be equated (since $\max _{\eta}=\max _{\theta}=\min _{\eta}=\min _{\theta}=5$ ), channels $\zeta$ and $\xi$ would be equated (since $\max _{\zeta}=\max _{\xi}=5$ and $\min _{\zeta}=\min _{\xi}=4$ ), but the last two ones would not be equivalent to the first two ones, as desired.

Also in this case, this refined notion of equivalence has a well-known counterpart in concurrency theory: it corresponds to what is usually called (complete) 
trace equivalence [1]. A complete trace (or, simply, a trace) for a channel $\eta$ is a sequence $\left\langle n_{1} \ldots n_{k}\right\rangle$ such that there exist transitions $\eta_{0} \stackrel{n_{1}}{\longrightarrow} \eta_{1} \ldots \stackrel{n_{k}}{\longrightarrow} \eta_{k}$ where $\eta_{0}=\eta$ and $\eta_{k}$ is dead. We denote by $\operatorname{tr}(\eta)$ the set of complete traces of a channel $\eta$. Two channels are complete trace equivalent (or, simply, trace equivalent) if they have identical sets of complete traces.

To prove this characterization (that is the main theoretical result of our paper), we use some classical definitions and results from the theory of network flows. The key result is Lemma 2. To prove it, we find it useful to work in a framework where values are associated with edges and flows are expressed by assigning a flow to every edge (and not to every path). Graphs where vertices are weighted can be easily transformed in "equivalent" graphs where edges are weighted by applying a well-known node splitting technique $[2,2.4]$ : a node $v$ with capacity $x$ is split into two nodes, $v_{\iota}$ and $v_{o}$, connected by an edge with capacity $x$. All inputs to $v$ are connected with $v_{\iota}$ and all outputs with $v_{o}$ through edges with infinite capacity. Moreover, the edge-oriented presentation of flows is less abstract than the path-oriented one, in that there may be more pathoriented flows corresponding to one edge-oriented [2, Theorem 3.5]. Thus, from now until Lemma 2 included, we assume that edges are weighted and flows are formulated in an edge-oriented way. To simplify reading, we still denote by $\eta$ and $\phi$ nets with weighted edges and edge-oriented flows.

Definition 1 (Cut). A cut $S$ is a subset of the vertices such that $s \in S$ and $t \notin S$. We denote by $S \rightarrow$ the set of edges $(u, v)$ such that $u \in S$ and $v \notin S$. The capacity of a cut $S$ is $\sum_{e \in S \rightarrow \eta} \eta(e)$. We say that $\phi$ saturates a cut $S$ of $\eta$ if, for every $e \in S^{\rightarrow}$, it holds that $\phi(e)=\eta(e)$.

Definition 2 (Residual net). Given a channel $(V, E, \eta)$ and a flow $\phi$, the residual net of $\eta$ after $\phi$ is $\left(V, E_{\phi}, \eta_{\phi}\right)$, where

$$
\eta_{\phi}(u, v) \triangleq \begin{cases}\eta(u, v)-\phi(u, v) & \text { for every }(u, v) \in E \\ \phi(v, u) & \text { for every }(v, u) \in E\end{cases}
$$

and $E_{\phi} \triangleq\left\{(u, v): \eta_{\phi}(u, v)>0\right\}$. Like before, we usually denote by $\eta_{\phi}$ the residual net of $\eta$ after $\phi$.

Definition 3 (Augmenting path). Given a channel $(V, E, \eta)$ and a flow $\phi$, an augmenting path for $\eta$ after $\phi$ is any path from $s$ to $t$ in $\eta_{\phi}$.

Theorem 2 (Maximum flow [2]). The value of $\phi$ is $\max _{\eta}$ if and only if there is no augmenting path in $\eta_{\phi}$.

Proposition 3 (Augmented flow [8]). Given a channel $\eta$, a flow $\phi$ of value $n<\max _{\eta}$ and an augmenting path $p$, the function $\phi^{\prime}: E \rightarrow \mathbb{N}$ defined by

$$
\phi^{\prime}(u, v) \triangleq \begin{cases}\phi(u, v)+1 & \text { if }(u, v) \in p \\ \phi(u, v)-1 & \text { if }(v, u) \in p \\ \phi(u, v) & \text { otherwise }\end{cases}
$$

is a flow for $\eta$ of value $n+1$. 
Proposition 4. $\phi$ inhibits $\eta$ if and only if it saturates at least one cut of $\eta$.

Proof. Trivial, by definition of cut, saturation and inhibition.

Definition 4. Given a vertex $u$ and a set of edges $K$, we write $u \triangleleft K$ to mean that every walk $u \rightsquigarrow t$ includes at least one edge of $K$.

Proposition 5. Let $\eta$ be a channel and $S$ one of its cuts. If $u \in S$, then $u \triangleleft S \rightarrow$.

Proof. Let $p$ be a walk $u=u_{0}, u_{1}, \ldots, u_{k}=t$, for $k>0$ (since $t \neq u$, because $u \in S$ and $t \notin S)$. Since $u \in S$ and $t \notin S$, there must exists an $i \in\{0, \ldots, k-1\}$ such that $u_{i} \in S$ and $u_{i+1} \notin S$; thus, $\left(u_{i}, u_{i+1}\right) \in S \rightarrow$.

Proposition 6. If $u \triangleleft K$ and $v \nless K$, then every walk from $u$ to $v$ includes at least one edge of $K$.

Proof. By contradiction, assume that there is a walk $u \stackrel{p}{\rightsquigarrow} v$ such that $p \cap K=\emptyset$. Take a walk $v \stackrel{q}{\sim} t$ such that $q \cap K=\emptyset$ (such a $q$ must exist because $v \nless K$ ). Then, $u \stackrel{p}{\rightsquigarrow} v \stackrel{q}{\sim} t$ would be a walk from $u$ to $t$ without edges in $K$, in contradiction with $u \triangleleft K$.

Proposition 7. Let $\eta$ be a channel and $\phi$ a flow that saturates one of its cuts $S$. Assume that there exists a $v \notin S$ and a non-empty $K \subseteq E$ such that $v \triangleleft K$ and all the edges in $K$ are saturated by $\phi$. Then, there exists a cut of $\eta$ greater (w.r.t. ' $\subseteq$ ') than $S$ still saturated by $\phi$.

Proof. Consider the set $P_{K}^{v} \triangleq\{x: x \triangleleft K \wedge v \rightsquigarrow x$ in $\eta\}$. We have that:

1. $P_{K}^{v} \nsubseteq S$, because $v \in P_{K}^{v}$ and $v \notin S$;

2. $S \cup P_{K}^{v}$ includes $s$ (because $s \in S$ ) and does not include $t$ (since $t \notin S$ and $t \notin P_{K}^{v}$, because $t \nless K$ being $\left.K \neq \emptyset\right)$;

3. every $(x, y) \in S \cup P_{K}^{\rightarrow}$ can be either

$x \in S$ and $y \in \overline{S \cup P_{K}^{v}}\left(=\bar{S} \cap \overline{P_{K}^{v}}\right)$ : in this case, $(x, y) \in S^{\rightarrow}$ and so it is saturated by $\phi$;

$x \in P_{K}$ and $y \in \overline{S \cup P_{K}^{v}}\left(=\bar{S} \cap \overline{P_{K}^{v}}\right):$ in this case, it must be that $y \nless K$, since $v \rightsquigarrow x$ (because $\left.x \in P_{K}^{v}\right)$ and $(x, y) \in E$. Then, by Proposition 6 , $(x, y) \in K$ and, hence, it is saturated by $\phi$.

Thus, $S \cup P_{K}$ would be a cut (by point 2 above) saturated by $\phi$ (by point 3 above) and strictly greater than $S$ (by point 1 above).

Definition 5 (Crossing a cut). Let $\eta$ be a channel, $S$ one of its cuts and $\phi$ a flow for it. An edge $(u, v) \in E_{\phi}$ crosses $S$ if, within $\eta$, it holds that $u \triangleleft S \rightarrow$ and $v \nless S^{\rightarrow}$. We say that an augmenting path crosses a cut if it includes at least one edge that crosses that cut.

Proposition 8. Let $\eta$ be a channel, $S$ one of its cuts and $\phi$ a flow for $\eta$. If $e$ crosses $S$, then either $e \in S \rightarrow$ or $e \in E_{\phi} \backslash E$. 
Proof. Let $e=(u, v)$; if $e \in E$, then it must be $e \in S^{\rightarrow}$, otherwise $v \rtimes S^{\rightarrow}$ would imply that $u \pitchfork S^{\rightarrow}$.

Proposition 9. Let $\eta$ be a channel and $\phi$ a flow. Every augmenting path crosses every cut of $\eta$ at least once.

Proof. Let $S$ be a cut of $\eta$ and $p$ be an augmenting path $s=u_{0}, \ldots, u_{k}=t$, for $k>0$. By Proposition 5, in $\eta$ we have that $s \triangleleft S^{\rightarrow}$; by Definition $4, t 丸 S \rightarrow$, since there exists a walk from $t$ to $t$ (viz., the empty path) that does not include any edge from $S \rightarrow$ (actually, it does not include any edge). Thus, there must be an $i \in\{0, \ldots, k-1\}$ such that $u_{i} \triangleleft S^{\rightarrow}$ and $u_{i+1} \Varangle S^{\rightarrow}$ in $\eta$, as required.

Lemma 2. Let $\eta$ be a channel and $\phi$ an inhibiting flow of value $n<\max _{\eta}$; then, there exists an inhibiting flow of value $n+1$.

Proof. Since $\phi$ inhibits the channel, by Proposition 4 there must be a cut of $\eta$ saturated by $\phi$; let us consider all such cuts and let $S$ be a maximal cut (w.r.t. ' $\subseteq$ '). Since the value of $\phi$ is smaller than $\max _{\eta}$, by Theorem 2 there exists an augmenting path for $\eta$ after $\phi$. We now prove the following claim:

There exists an augmenting path $p^{\prime}$ that crosses $S$ exactly once.

Proof. By Proposition 9, every augmenting path crosses $S$ at least once. Let us fix one of them, say $p$, and let $(u, v)$ be the first edge in $p$ that crosses $S$. There must be a walk $v \stackrel{q}{\sim} t$ in $\eta$ after $\phi$, otherwise $S$ would not be maximal. Indeed, by contradiction, assume that every walk from $v$ to $t$ in $\eta$ after $\phi$ includes at least one edge saturated by $\phi$. Let $K(\subseteq E)$ be the (non-empty) set of all such edges. By construction, $v \triangleleft K$; moreover, by the contrapositive of Proposition $5, v \pitchfork S \rightarrow$ entails that $v \notin S$. Thus, by Proposition 7, we could contradict maximality of $S$.

To conclude, we have that $p^{\prime} \triangleq s \stackrel{p}{\rightsquigarrow} u, v \stackrel{q}{\sim} t$ is an augmenting path with exactly one edge crossing $S$, viz. $(u, v)$. Indeed, $p^{\prime}$ is an augmenting path since $s \stackrel{p}{\sim} u, v$ is the initial portion of the augmenting path $p$ and $v \stackrel{q}{\sim} t$ only includes edges of $E$ with positive residual capacity (thus, belonging to $\eta_{\phi}$ ). Moreover, it only crosses $S$ once since both $s \stackrel{p}{\sim} u$ and $v \stackrel{q}{\sim} t$ do not include crossing edges: by construction, $(u, v)$ is the first of such edges in $p ; v \stackrel{q}{\sim} t$ is formed only by edges belonging to $E$ with a positive residual capacity (that, thus, cannot belong to $S^{\rightarrow}$ ) and, hence, cannot cross $S$, by Proposition 8 .

Let $\phi^{\prime}$ be the flow obtained by updating $\phi$ with $p^{\prime}$ as described in Proposition 3; if we prove that $\phi^{\prime}$ inhibits the channel, we have done. To this aim, by Proposition 4 it suffices to prove that it saturates $S$. If it was not the case, $p^{\prime}$ would include $(v, u)$, for some $(u, v) \in S^{\rightarrow}$. By Proposition $5, u \triangleleft S^{\rightarrow}$, since $u \in S$; hence, $(v, u)$ cannot be the edge of $p^{\prime}$ that crosses $S$. Then, it can either be $v \triangleleft S^{\rightarrow}$ or $v \rtimes S^{\rightarrow}$; however, both these possibilities lead to a contradiction (and this suffices to conclude): 
$v \nless S^{\rightarrow}$ : since (by Proposition 5) $s \triangleleft S^{\rightarrow}, v \nless S^{\rightarrow}$ implies that there must be an edge crossing $S$ before $(v, u)$ in $p^{\prime}$; since (by Definition 4) $t \nless S^{\rightarrow}, u \triangleleft S^{\rightarrow}$ implies that there must be an edge crossing $S$ after $(v, u)$ in $p^{\prime}$; since $p^{\prime}$ has only one edge crossing $S$, this case is not possible.

$v \triangleleft S \rightarrow$ : in this case, by Proposition 7 , we could exhibit a cut saturated by $\phi$ greater than $S$.

Remark 1. Given a channel $\eta$, we denote by $\hat{\eta}$ the net after the node splitting; given a path-oriented flow $\phi$, we denote by $\check{\phi}$ the corresponding edge-oriented flow. It is easy to see that $\eta$ and $\hat{\eta}$ have the same maximum flow; moreover, any inhibiting flow for $\eta$ can be turned into an inhibiting flow for $\hat{\eta}$, and vice versa.

If we have an inhibiting flow $\phi$ of value $n$ for $\eta$, we can define an edge-oriented inhibiting flow $\check{\phi}$ for $\hat{\eta}$ of value $n$. By Lemma 2, we can obtain an inhibiting flow $\check{\phi}^{\prime}$ for $\hat{\eta}$ of value $n+1$ and then turn it into inhibiting flow $\phi^{\prime}$ for $\eta$ of value $n+1$ (flow are transformed by following [2, Theorem 3.5]).

Theorem 3. Two channels are trace equivalent if and only if they have identical maximum and minimum inhibiting flow.

Proof. (If) Let $\left\langle n_{1} \ldots n_{k}\right\rangle \in \operatorname{tr}(\eta)$; because of Proposition 1, there exists an inhibiting transition for $\eta$ with value $n=n_{1}+\ldots+n_{k}$. If $n \in\left\{\min _{\eta}, \max _{\eta}\right\}$, by hypothesis we have that there exists an inhibiting transition for $\theta$ with value $n$; otherwise, we can start from a minimum inhibiting flow and use Remark 1 for $n-\min _{\eta}$ times to obtain an inhibiting flow for $\theta$ with value $n$. In both cases, by Proposition 2 we have that $\left\langle n_{1} \ldots n_{k}\right\rangle \in \operatorname{tr}(\theta)$, as desired.

(Only if) Let us consider the traces $\left\langle\min _{\eta}\right\rangle$ and $\left\langle\max _{\eta}\right\rangle$, both belonging to $\operatorname{tr}(\eta)$; by hypothesis, they also belong to $\operatorname{tr}(\theta)$. If by contradiction were $\left.\max _{\theta}\right\rangle$ $\max _{\eta}$ (it cannot be ' $<$ ' because $\left\langle\max _{\eta}\right\rangle \in \operatorname{tr}(\theta)$ ), we would have that $\left\langle\max _{\theta}\right\rangle \in$ $\operatorname{tr}(\theta)$ but $\left\langle\max _{\theta}\right\rangle \notin \operatorname{tr}(\eta)$, in contradiction with $\operatorname{tr}(\eta)=\operatorname{tr}(\theta)$. If $\min _{\theta}<\min _{\eta}$, the proof is similar. Thus, $\max _{\theta}=\max _{\eta}$ and $\min _{\theta}=\min _{\eta}$, as desired.

Notice that every channel is trace equivalent to a channel, that we call canonical, with a very simple topology (in particular, it has no cycles). Let us define the channel $\gamma_{m, n}$ as:
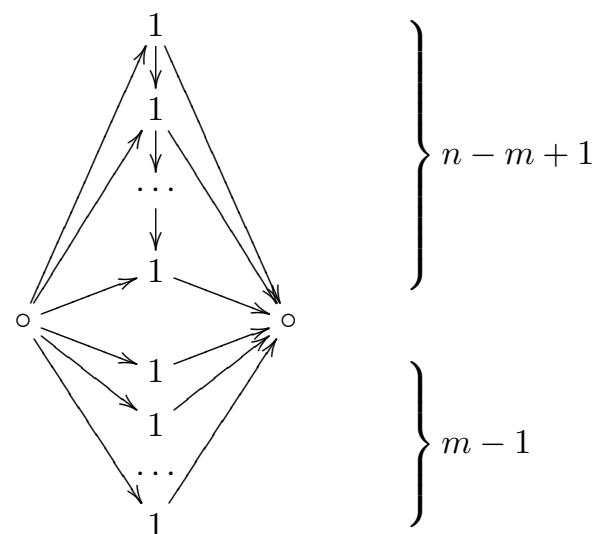
It is easy to check that $\min _{\gamma_{m, n}}=m$ and $\max _{\gamma_{m, n}}=n$. Thus, $\gamma_{m, n}$ can be considered the standard representative of the trace-equivalence class of all the nets with minimum inhibiting flow $m$ and maximum flow $n$.

\section{Complexity Issues}

Theorem 3 characterizes trace equivalence in terms of maximum flow and minimum inhibiting flow. It is well-known that there exist polynomial time algorithms for finding the maximum flow in a net. We are left with studying the complexity of the following problem, that we call minimum inhibiting flow (MIF, for short):

MIF: Given a network $\eta$, find the value of the minimum inhibiting flow for $\eta$.

MIF can be turned into a decisional problem:

DMIF: Given a network $\eta$ and an integer $k$, is there an inhibiting flow for $\eta$ with value at most $k$ ?

Theorem 4. MIF is NP-complete.

Proof. Clearly, DMIF is in NP; we now prove that also MIF is in NP. First of all, notice that the size of a channel $(V, E, \eta)$ is $O(|E|+|V| \log C)$, where $C=\max _{v \in V} \eta(v)$; moreover, $\max _{\eta} \in O(|V| C)$. Since DMIF is in NP, we have a polynomial verifier for it. Thus, since $\min _{\eta} \in\left\{1, \ldots, \max _{\eta}\right\}$, we can use a binary search and the polynomial verifier for DMIF to find the exact value of $\min _{\eta}$ in polynomial time.

To show that MIF is NP-hard, we reduce the problem of finding a maximal matching of a given cardinality in a bipartite graph to DMIF. We recall that a maximal matching in a graph $(V, E)$ is a set of edges $F \subseteq E$ such that:

$-\forall e, e^{\prime} \in F$ it holds that $e \cap e^{\prime}=\varnothing ;$

- $\forall e \in E \exists e^{\prime} \in F$ such that $e \cap e^{\prime} \neq \varnothing$.

Let $G=\left(V_{1}, V_{2}, E\right)$ be a bipartite undirected graph. We consider the channel $\left(V^{\prime}, E^{\prime}, \eta\right)$, where

$-V^{\prime}=V_{1} \cup V_{2} \cup\{s, t\}$, for $\{s, t\} \cap\left(V_{1} \cup V_{2}\right)=\varnothing$;

- $E^{\prime}=\left\{(u, v):\{u, v\} \in E \wedge u \in V_{1} \wedge v \in V_{2}\right\} \cup \bigcup_{u \in V_{1}}\{(s, u)\} \cup \bigcup_{u \in V_{2}}\{(u, t)\}$;

- $\eta(v)=1$ for every $v \in V_{1} \cup V_{2}$.

We now show that $G$ has a maximal matching of cardinality $k$ if and only if $\eta$ has an inhibiting flow of value $k$.

(If). Assume that $\phi$ is an inhibiting flow of value $k$ for $\eta$ and let $M=\{\{u, v\}$ : $\left.u \in V_{1} \wedge v \in V_{2} \wedge \phi((u, v)) \neq 0\right\}$. We now prove that $M$ is a maximal matching for $G$ of cardinality $k$. If there were an edge $(u, v) \in E$ whose endpoints are not covered by any edge in $M$, then the charge of $u$ and $v$ would still be 1 after $\phi$ and suvt would be a legal path in $\eta$ after $\phi$. Moreover, by construction 
of $\eta$, there exist $u_{1}, \ldots, u_{k} \in V_{1}$ such that $\phi\left(\left(s, u_{i}\right)\right)=1$ for every $i$; then, by definition of flow and construction of $\eta$, for every $i=1, \ldots, k$ there exists $v_{i} \in V_{2}$ such that $\phi\left(\left(u_{i}, v_{i}\right)\right)=1$, and $v_{i} \neq v_{j}$ whenever $i \neq j$. Thus, $M$ is a maximal matching of cardinality $k$ in $G$.

(Only if). Let $M$ be a maximal matching in $G$ and consider the function $\phi$ : $E^{\prime} \rightarrow \mathbb{N}$ such that

$$
\phi((s, u))=\phi((u, v))=\phi((v, t))=1
$$

for every $\{u, v\} \in M$, with $u \in V_{1}$ and $v \in V_{2}$. Clearly, $\phi$ is a flow for $\eta$ of value $k$. Moreover, it inhibits $\eta$. If it were not the case, there should be a path $s u v t$ in $\eta$ after $\phi$; this implies, by definition of path, that the charge of $u$ and $v$ has not been consumed by $\phi$ that, hence, would not touch $u$ and $v$. But then $M$ is not a maximal matching for $G$ since the edge $\{u, v\} \in E$ has endpoints not covered by any edge of $M$.

We observe that, in the reduction just shown, we need to consider acyclic unitary networks only, i.e. networks in which depletable charge of each node is 1. This implies that MIF is an intractable problem even in this restricted case.

It is now worth noting that in concurrency theory complexity measures are usually expressed in terms of the size of the labeled transition system (LTS, for short) resulting from all the labeled transitions of a given process (in our case, a channel). This is because the definitions and characterizations of process equivalences are usually given on the LTSs of the equated processes, and not on the processes themselves. Even for simple models like CCS, trace equivalence is exponential in the size of the LTS [23], while other equivalences (like, e.g., bisimilarity $[19,16])$ are polynomial [12]. However, if expressed in terms of the size of the process, all these equivalences become (at least) exponential, since the number of states of a LTS is exponential in the size of its originating process.

Thanks to Theorem 3, we could have directly checked equivalences on the LTSs resulting from the equated channels. However, also in our case we would have an exponential blow up of the number of states. For example, consider the channel:

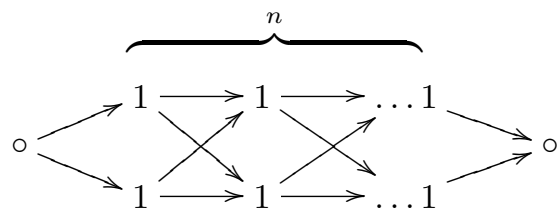

It has $2 n+2$ vertices and it produces a LTS with $2^{n}$ states: there are $2^{n}$ paths along which a unitary flow can be sent.

We have instead defined our behavioural equivalence by relying on properties of the equated channels, and not of their LTSs. Nevertheless, as we have just shown, trace equivalence seems not verifiable in polynomial time (w.r.t. the size of the equated channels); this should not be surprising. On one hand, this agrees with the usual hardness of trace equivalence in concurrency theory mentioned above; on the other hand, this stimulates future work on more efficiently verifiable, but still properly discriminating, equivalences. 


\section{Beyond Trace Equivalence}

To conclude our presentation of trace equivalence, let us pinpoint some of its limitations; such issues are standard in concurrency theory and scales to our model too. The main issue is that trace equivalence is not preserved by transitions. Indeed, consider the nets $\eta$ and the canonical net $\gamma_{2,2 n}$, for any $n>1$ :
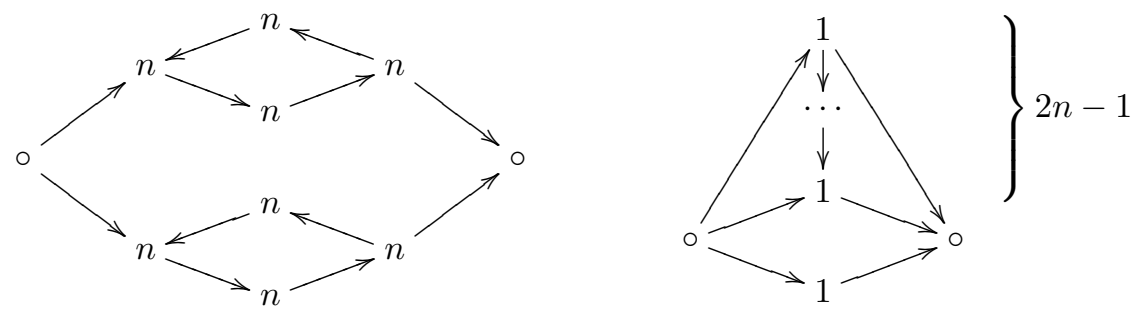

Clearly, they are trace equivalent. Then, consider the unitary transition of $\eta$ that inhibits its norther cycle by taking $n$ times such a cycle; it turns $\eta$ into the channel $\eta^{\prime}$ having 0 on every node of its northern cycle. There is no unitary transition of $\gamma_{2,2 n}$ that leads to a channel that is trace equivalent to $\eta^{\prime}$. Indeed, if the unitary flow passes through the bottom vertex, the resulting channel has a maximum flow of $2 n-1$ (whereas $\max _{\eta^{\prime}}=n$ ); if the flow passes through (some of) the top $2 n-1$ vertices, the resulting channel has a minimum inhibiting flow greater than 1 (whereas $\min _{\eta^{\prime}}=1$ ).

In concurrency theory, a classical notion of equivalence that is more finely grained than those based on traces relies on the notion of bisimulation. In our framework, this a symmetric relation $\Re$ on channels such that $\eta \Re \theta$ and $\eta \stackrel{n}{\longrightarrow} \eta^{\prime}$ imply $\theta \stackrel{n}{\rightarrow} \theta^{\prime}$, for some $\theta^{\prime}$ such that $\eta^{\prime} \Re \theta^{\prime}$. Two channels $\eta$ and $\theta$ are called bisimulation equivalent if they are related by a bisimulation. In view of Theorem 3 , it follows immediately from the definition that bisimulation equivalence is included in trace equivalence. Moreover, by what we have just said, the inclusion is strict: the channels $\eta$ and $\gamma_{2,2 n}$ depicted above are not bisimulation equivalent. Another example is given by channels $\zeta$ and $\xi$ of Figure 1: after sending two items along $\circ 524 \circ$ in $\zeta$ we have a net with maximum flow at 2 ; on the contrary, every 2 -valued flow in $\xi$ yields a channel with maximum flow greater than 2 .

A challenging issue for future work is finding a characterization of bisimulation equivalence in terms of structural properties of channels, in the same spirit as the characterizations we have provided for trace equivalence in this paper.

\section{Conclusions and Related Work}

We presented a simple model of communication networks, called channels. The communication infrastructure is modeled by a graph connecting a sender $s$ with a receiver $t$. Nodes have a depletable charge. Labeled transitions are used to 
describe the dynamics of channels, where states of the LTS are channels of identical topology and labels are the number of information units transmitted in a communication from $s$ to $t$ via a legal network flow. We equated channels by means of intrinsic channel properties (that is, their maximum flow and minimum inhibiting flow) and studied their complexity. Finally, we showed that such equivalence coincides with a natural notion of equivalence borrowed from concurrency theory.

There are several research lines that can be pursued to develop the framework presented in this paper. First of all, we assume that source and target are fixed during a channel evolution. More realistic models include scenarios where only the target is fixed (e.g., sensor networks) or where both source and target can be any node of the net. Moreover, our model assumes that the network topology does never change during the computation. This is clearly a simplifying assumption and makes our model unsuited for MANETs. It would be challenging to introduce in the model such advanced features and study the resulting equivalences.

Related work. In the last years, network scenarios have been modeled and studied by means of process algebraic techniques. In such papers, the authors usually first give a syntax for writing nets, featuring some distinguishing issues of the modeled applications; then, they give an operational semantics and a behavioural equivalence to reason over nets; finally, the theory is used in some concrete application, e.g. to verify the correctness of some network protocol or to equate different networks with the same behaviour. According to the kind of network modeled, we mention: $[11,13,22]$, where mobile ad hoc networks are considered; $[15,17,14]$, where wireless systems are considered; [5], where peer-topeer overlay networks are considered. Our approach clearly follows this research line. However, we do not have a process syntax and just write networks via their physical topology, assuming that some suitable software is hardcoded into every node of the net to properly implement some routing strategy. A somehow similar approach has been followed by some of the authors in a previous paper [7], where the framework was based on (hyper)graph rewriting. There, apart from functional equivalence, other network measures (e.g., robustness) were related to bisimulation in the model.

It is worth saying that our MIF problem somehow resembles the Network Inhibition Problem (NIP) [20]. There, every edge of a flow net is equipped with a destruction cost; the problem is to find a flow that leaves the net in the worst possible condition (i.e., with the minimum max flow) and whose cost is smaller than a given quantity. In loc. cit., it is proved that NIP is NP-complete for several class of graphs, but polynomially approximable for most of them (e.g., planar or grid).

A related paper is [21], where a network model (somehow similar to ours) is used to study the complexity of finding optimal flow subnetworks. A challenging issue for future research is the understanding of how the two approaches relate to each other. 
To conclude, we have proposed a usage of formal models different from those usually exploited in the network community. There, formal models are often used [9] for model checking and simulations to study, e.g., correctness of network protocols, optimal schedulings, network measures or power consumption.

Acknowledgements Thanks to Flavio Chierichetti for his valuable support in the proof of Theorem 4.

\section{References}

1. L. Aceto, W. Fokkink, and C. Verhoef. Structural operational semantics. In J. Bergstra, A. Ponse, and S. Smolka, editors, Handobook of Process Algebra, pages 197-292. North-Holland, 2001.

2. R. Ahuja, T. Magnanti, and J. Orlin. Network Flows, theory, algorithms, and applications. Prentice-Hall, New Jersey, 1993.

3. K. Akkaya and M. F. Younis. A survey on routing protocols for wireless sensor networks. Ad Hoc Networks, 3(3):325-349, 2005.

4. I. Akyildiz, W. Su, Y. Sankarasubramaniam, and E. Cayirci. A survey on sensor networks. IEEE Communications Magazine, 40(8):102-116, 2002.

5. J. Borgström, U. Nestmann, L. O. Alima, and D. Gurov. Verifying a structured peer-to-peer overlay network: The static case. In Global Computing, volume 3267 of $L N C S$, pages 250-265. Springer, 2005.

6. A. Boukerche, editor. Algorithms and Protocols for Wireless Sensor Networks. Wiley \& Sons, 2009.

7. P. Cenciarelli, D. Gorla, and E. Tuosto. Network applications of graph bisimulation. In R. Heckel and G. Taentzer, editors, Proceedings of the 4th International Conference on Graph Transformation (ICGT'08), volume 5214 of LNCS, pages 131-146. Springer, 2008.

8. T. Cormen, C. Leiserson, and R. Rivest. Introduction to Algorithms. MIT Press, 1990.

9. A. Fehnker and A. McIver. Formal techniques for the analysis of wireless networks. In 2nd International Symposium on Leveraging of Formal Maethods, Verification and Validation (IEEE-ISOLA). IEEE, 2006.

10. Y. Ganjali and A. Keshavarzian. Load balancing in ad hoc networks: single-path routing vs. multi-path routing. In INFOCOM 2004. Twenty-third Annual Joint Conference of the IEEE Computer and Communications Societies, volume 2, pages 1120-1125, 2004

11. J. C. Godskesen. A calculus for mobile ad hoc networks. In Proc. of COORDINATION, volume 4467 of LNCS, pages 132-150. Springer, 2007.

12. P. Kanellakis and S. Smolka. CCS Expressions, Finite State Processes and Three Problems of Equivalence. Information and Conputation, 86(1):43-68, 1990. An extended abstract appeared in Proc. of ACM PODC'83.

13. M. Merro. An observational theory for mobile ad hoc networks. In Proc. of MFPS, volume 173 of ENTCS, pages 275-293, 2007. Full version to appear in Information and Computation.

14. M. Merro and E. Sibilio. A timed calculus for wireless networks. In Proc. of FSEN, 2009. To appear.

15. N. Mezzetti and D. Sangiorgi. Towards a calculus for wireless systems. In Proc. of MFPS, volume 158 of ENTCS, pages 331-353, 2006. 
16. R. Milner. Communication and Concurrency. Prentice Hall, 1989.

17. S. Nanz and C. Hankin. A framework for security analysis of mobile wireless networks. Theoretical Computer Science, 367(1-2):203-227, 2006.

18. N. Nehra, R.B.Patel, and V.K.Bhat. Routing with load balancing in ad hoc network: A mobile agent approach. In 6th IEEE/ACIS International Conference on Computer and Information Science (ICIS 2007), 2007.

19. D. Park. Concurrency and automata on infinite sequences. In Theoretical Computer Science, volume 104 of LNCS, pages 167-183. Springer, 1981.

20. C. A. Phillips. The network inhibition problem. In Proc. of STOC, pages 776-785. ACM Press, 1993.

21. T. Roughgarden. On the severity of braess's paradox: Designing networks for selfish users is hard. Journal of Computer and System Science, 72(5):922-953, 2006.

22. A. Singh, C. Ramakrishnan, and S. A. Smolka. A process calculus for mobile ad hoc networks. In Proc. of COORDINATION, volume 5052 of $L N C S$, pages 296-314. Springer, 2008.

23. L. Stockmeyer and A. Meyer. Word Problems Requiring Exponential Time. In Proc. of 5th Symp. on Theory of Computing (STOC), pages 1-9. ACM, 1973.

24. C. Toh. Ad Hoc Mobile Wireless Networks: Protocols and Systems. Prentice Hall, 2002.

25. O. K. Tonguz and G. Ferrari. Ad Hoc Wireless Networks: A CommunicationTheoretic Perspective. John Wiley \& Sons, 2006. 